\title{
A population-based study of the use of radium 223 in metastatic castration-resistant prostate cancer: Factors associated with treatment completion
}

\author{
Sunil Parimi, $M D^{\prime}$; Erica Tsang, $M D^{2}$; Abraham Alexander, $M D^{3} ;$ Michael McKenzie, $M D^{3}$; \\ Francois Bachand, MD; Katherine Sunderland, MD'; Kim N. Chi, MD'; Maria Aparicio, MD'; \\ Daniel Worsley, MDi; Scott Tyldesley, $M D^{3}$
}

'Medical Oncology, British Columbia Cancer Agency; ${ }^{2}$ Internal Medicine, University of British Columbia; ${ }^{3}$ Radiation Oncology, British Columbia Cancer Agency; ${ }^{4}$ Genitourinary Cancer Outcomes Unit, British Columbia Cancer Agency; ${ }^{5}$ Nuclear Medicine, Vancouver General Hospital; Vancouver, BC, Canada

Cite as: Can Urol Assoc J 2017;11 (10):350-5. http://dx.doi.org/10.5489/cuaj.4415

\section{Abstract}

Introduction: Radium 223 (Ra223) given for six cycles has proven efficacy in clinical trials, but its population-level generalizability has not been well-described. The objectives of this study were to describe population-based Ra223 use in the abiraterone and enzalutamide era and identify factors associated with completion. Methods: All Ra223 patients at the British Columbia Cancer Agency between September 2013 and February 2016 were identified. Patients who completed $<5$ vs. $\geq 5$ cycles were compared on patient characteristics, lines of prior therapy, prostate-specific antigen (PSA) and alkaline phosphatase (ALP) decline $>30 \%$ from baseline (R30\%), and survival, to identify factors associated with therapy completion.

Results: Ninety-one patients were identified; 48 (52.7\%) completed $\geq 5$ cycles. Median overall survival (mOS) was 10.7 months, PSA and ALP R30\% were $21 \%$ and $52 \%$, respectively. Completion of $<5$ cycles was associated with higher baseline ALP $(p=0.05)$ and lower baseline hemoglobin $(\mathrm{Hb})$ levels $(p=0.03)$. Patients in the $\geq 5$ cycles group had longer mOS than those in the $<5$ cycles group $(16.2$ vs. 5.9 months; $\mathrm{p}<0.0001)$, as well as higher PSA R30\% $(33.3 \%$ vs. 7.0\%; $p=0.002)$ and ALP R30\% (66.7\% vs. 34.9\%; $p=0.03)$. Patients with $\mathrm{ALP} \geq 220$ and $\mathrm{Hb} \leq 118$ had 3.85 times the odds of not completing $\geq 5$ cycles vs. ALP $<220$ and $\mathrm{Hb}>118$.

Conclusions: Compared to clinical trials, patients in a populationbased setting had more lines of therapy and shorter survival. Lower ALP and higher hemoglobin were associated with completion of $\geq 5$ cycles, longer mOS, and greater incidence of PSA and ALP response.

\section{Introduction}

The options for therapy in metastatic castration-resistant prostate cancer (mCRPC) have greatly expanded over the past decade. Palliative radiotherapy to painful sites of bone metastases continues to be commonly used to relieve symp- toms at progression of mCRPC. Targeted agents (e.g., abiraterone and enzalutamide) ${ }^{1-4}$ and chemotherapeutics (e.g., docetaxel and cabazitaxel $)^{5,6}$ are now mainstay options. Radiopharmaceuticals have also emerged as another viable option in this patient population.

Radioisotopes, such as the beta-emitter strontium 89 (Sr89), have been used in the past to decrease the rate of progression of new site of pain either on its own, or in combination with external beam radiotherapy. Radium 223 chloride (Ra223) is a newer radiopharmaceutical agent given as a monthly intravenous injection for six cycles. It is an alpha-emitting radioisotope that mimics calcium, and is taken up into newly formed bone. Alpha particles induce double-stranded DNA breaks in adjacent cells, with a very short penetration ( $<10$ cell diameters). As a result, Ra223 has the potential to cause a highly localized tumour cell killing immediately adjacent to areas of new bone formation, with very minimal marrow toxicity.

The definitive phase 3 trial (ALSYMPCA) ${ }^{7}$ evaluating Ra223 or placebo in $921 \mathrm{mCRPC}$ patients demonstrated an overall survival (OS) benefit of 3.5 months, with a median overall survival (mOS) of 14.9 months for Ra223 vs. 11.3 months for placebo (hazard ratio [HR] 0.70; 95\% confidence interval $[\mathrm{Cl}] 0.58-0.83 ; \mathrm{p}<0.001)$. Assessments of all main secondary efficacy endpoints also showed a benefit of Ra233 as compared to placebo. Prostate-specific antigen (PSA) response rate (defined as a $>30 \%$ decline from baseline) was $21 \%$ and alkaline phosphatase (ALP) response rate (defined as a decline $>30 \%$ ) was $52 \%$. In addition, Ra223 was associated with low myelosuppression rates and fewer adverse events. Toxicity was minimal, as overall and serious adverse events were lower in the Ra223 group compared to the placebo group. Grade 3-4 thrombocytopenia, a particular concern with other radioisotopes, was minimal (4\% with Ra223 vs. $2 \%$ with placebo), although diarrhea was more common with Ra223 ( $22 \%$ vs. $13 \%$ ), but no difference 
was seen in Grade 3 or 4 toxicity. In the ALSYMPCA trial, $72.8 \%$ of patients completed all six injections. ${ }^{7}$

In September 2013, Ra223 was made available for patients in British Columbia through two sequential access programs, and became publically funded in February 2015. Enzalutamide and abiraterone were also publically available during this time of Ra223 introduction, unlike during the time the ALSYMPCA trial accrued patients. We retrospectively sought to: 1) describe the use of Ra223, survival, PSA, and ALP response rate after Ra223 in a population-based setting in a contemporary era when abiraterone and enzalutamide were available; and 2) identify factors associated with successful completion of Ra223.

\section{Methods}

\section{Patient selection}

Those who had received their first cycle of Ra223 at the British Columbia Cancer Agency (BCCA) between September 2013 (when Ra223 first became available) and February 2016 were included in this analysis. Eligibility for Ra223 in the province was as summarized in the online protocol at bccancer.bc.ca based on the ALSYMPCA trial criteria and the product monogram. All approvals for Ra223 prescriptions were centralized through the provincial Compassionate Assess Program, which involved second physician and pharmacy check of eligibility before approval for funding. The BCCA was the sole provider of all Ra223, and all provincial utilization was captured in this analysis.

\section{Data collection and outcomes of interest}

Electronic records were retrospectively reviewed and abstracted clinical and laboratory factors were collected at baseline and prior to each Ra223 treatment. The maximum number of cycles of Ra223 completed by each patient was identified. Serum ALP and PSA were collected prior to each cycle, post-therapy completion, and at time of therapy discontinuation. In those patients who discontinued Ra223 prematurely, reasons for doing so were ascertained via progress notes. Progression was defined as deterioration in clinical status, PSA progression deemed by the treating physician to warrant change of therapy, or radiological progression defined according to the modified Response Evaluation Criteria in Solid Tumours Version 1.1 (RECIST v. 1.1). ${ }^{8}$ Although anemia and thrombocytopenia could be due to progression or drug-related effect, discontinuation for these reasons was scored as a drug-related side-effect for purposes of the analysis if disease progression was not otherwise described. The study was approved by the BC Cancer Agency Research Ethics Board.

\section{Study analysis}

Overall survival and followup time were calculated using the Kaplan-Meier method. PSA and ALP response rates were calculated as best response from baseline and categorized using two response thresholds: maximum decline in PSA from baseline $>30 \%$ and $>50 \%$ from the baseline value separately for both PSA and ALP.

Patient characteristics were described using frequencies and proportions for categorical variables, and with measures of central tendencies for numeric variables. Cases were binarized into those that completed $\geq 5$ cycles of Ra223 vs. those that completed $<5$ cycles. Abstracted clinical and biochemical factors were compared between these two groups to determine which were associated with completion of therapy. Chi square test or the Fisher's exact test, where appropriate, was used to analyze categorical variables between these two groups. Differences in medians were assessed using the two-sample median t-test.

PSA and ALP response rates were determined for the entire group and for comparison between those patients that completed $\geq 5$ cycles versus those that completed $<5$ cycles of Ra223. The odds of treatment completion were compared using logistic regression for three baseline ALP and hemoglobin combinations: a) higher-risk patients with high baseline $\operatorname{ALP}(\geq 220 \mathrm{IU} / \mathrm{L})$ and low hemoglobin $(\leq 118 \mathrm{~g} / \mathrm{L}) ; \mathrm{b})$ lowerrisk patients with lower baseline $\operatorname{ALP}(<220 \mathrm{IU} / \mathrm{L})$ and higher hemoglobin (>118 g/L); and c) intermediate-risk patients with baseline ALP $(<220 \mathrm{IU} / \mathrm{L})$ and hemoglobin $(\leq 118)$ or baseline ALP $(\geq 220 \mathrm{IU} / \mathrm{L})$ and hemoglobin $(>118)$. These cut points were selected a priori based on median values for hemoglobin in our population, and on previous reports of association with cycle completion for ALP. ${ }^{9,10}$

Median OS was ascertained for the entire cohort, and compared between groups completing 5 vs. $<5$ cycles using log rank test. Patients that were lost to followup were censored with the assumption of non-informative censoring.

Statistical analyses were performed in SAS 9.2 (SAS Institute Inc., Cary, NC, U.S.).

\section{Results}

Ninety-one patients were included in this study and their baseline characteristics are described in Table 1. Relative to patients enrolled on the ALSYMPCA trial, the patients treated in this population-based cohort appeared to be older (median of 73.9 years in this cohort vs. 71 years in ALSYMPCA), had worse Eastern Cooperative Oncology Group (ECOG) performance status (i.e., ECOG performance status $\geq 2$ 29.7\% vs. $13 \%$ in ALSYMPCA), and had more lines of therapy for CRPC (including abiraterone, enzalutamide, docetaxel, carbazitaxel, mitoxanthrone, or an experimental therapy). In the present series, the $71 \%$ of the patients had two or 


\begin{tabular}{|c|c|c|c|c|c|}
\hline Baseline clinical characteristics & All patients ${ }^{a}$ & $<5$ cycles $^{b}$ & $\geq 5$ cycles $^{\mathrm{c}}$ & $\begin{array}{c}P \text { comparing } \\
>5 \text { vs. }<5 \text { cycles }\end{array}$ & $\begin{array}{c}\text { Patients on } \\
\text { ALSYMPCA } \\
{\text { receiving } R a 223^{d}}^{d}\end{array}$ \\
\hline Age, median (range) & $73.9(50.0-94.0)$ & $71.5(57.0-91.3)$ & $72.5(50.0-94.0)$ & 0.17 & $71(49-90)$ \\
\hline Ra223 cycles, median (range) & $5(1-6)$ & $3(1-4)$ & $6(5-6)$ & $<0.0001$ & 6 \\
\hline ECOG performance status $>2, \mathrm{n}(\%)$ & $27(29.7)$ & $16(37.2)$ & 11 (22.9) & 0.14 & $77(13)$ \\
\hline Patients with $>6$ bone metastases, $n(\%)$ & $80(87.9)$ & $40(93.0)$ & $40(83.3)$ & 0.16 & $511(84)$ \\
\hline Patients with nodal metastases, n (\%) & $30(33.0)$ & $14(32.6)$ & $16(33.3)$ & 0.94 & $\mathrm{~N} / \mathrm{A}$ \\
\hline \multicolumn{6}{|l|}{ Laboratory values } \\
\hline Hemoglobin, median (range) (g/L) & $118(92-167)$ & $114(95-167)$ & $120(92-143)$ & 0.03 & $122(85-157)$ \\
\hline ALP, median (range) (IU/L) & $148(27-3440)$ & $210(68-3440)$ & $131.5(27-713)$ & 0.05 & $211(32-6431)$ \\
\hline Albumin, median (range) ( $\mathrm{g} / \mathrm{L}$ ) & 39 (27-99) & 38 (27-99) & $39(30-44)$ & 0.19 & $40(24-53)$ \\
\hline $\begin{array}{l}\text { Lactate dehydrogenase, median (range) } \\
\text { (IU/L) }\end{array}$ & $296(68-958)$ & $295(68-958)$ & $304(136-567)$ & 0.29 & $315(76-2171)$ \\
\hline PSA (ug/L), median (range) & $112.2(0.4-2800)$ & $137.28(0.96-2800)$ & $102.1(0.4-1662.2)$ & 0.76 & $146(3.8-6026)$ \\
\hline $\begin{array}{l}\text { Prior systemic treatments for CRPC, } \\
\text { median (range) }\end{array}$ & $2(0-5)$ & $2(0-5)$ & $2(0-5)$ & 0.21 & NR \\
\hline $\begin{array}{l}\text { Median number of systemic treatments } \\
\text { (range) }\end{array}$ & $2(0-5)$ & $2(0-5)$ & $2(0-5)$ & 0.21 & NR \\
\hline Previous docetaxel, n (\%) & $47(57.7)$ & $25(58.1)$ & $22(45.8)$ & 0.24 & $352(57.3)$ \\
\hline Previous abiraterone, $\mathrm{n}(\%)$ & $60(65.9)$ & $31(72.1)$ & $29(60.4)$ & 0.24 & 0 \\
\hline Previous enzalutamide, n (\%) & $77(84.6)$ & $36(83.7)$ & $41(85.4)$ & 0.82 & 0 \\
\hline Previous cabazitaxel, n (\%) & $4(4.4)$ & $1(2.3)$ & $3(6.3)$ & 0.62 & NR \\
\hline Previous clinical trial, n (\%) & $23(25.3)$ & $12(27.9)$ & $11(22.9)$ & 0.58 & $N / A$ \\
\hline
\end{tabular}

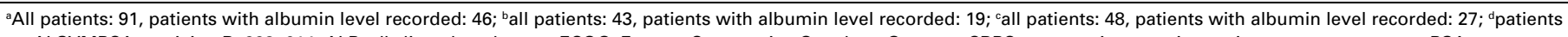

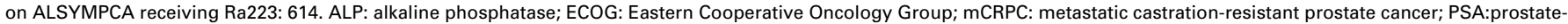
specific antigen.

more, and some (3\%) up to five, prior lines of such therapy; whereas in the ALSYMPCA study, $57 \%$ of patients had prior docetaxel and none had prior abiraterone or enzalutamide.

Median followup time was 10.3 months and all cases started their first cycle of Ra223 at least six months before analysis. The mOS was 10.7 months (95\% Cl 9.2-12.7) (Fig. 1). PSA response rate for a $>30 \%$ decline from baseline was $21 \%$, and PSA response rate for a $>50 \%$ decline from

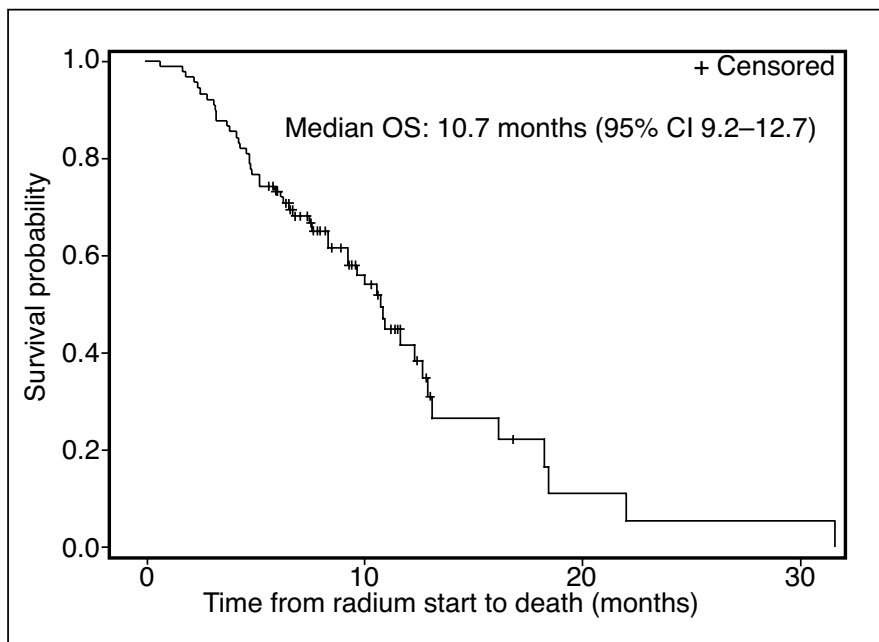

Fig. 1. Median overall survival (OS) for all patients on Ra223. Cl: confidence interval. baseline was $11 \%$. ALP response rate for $>30 \%$ decline was $52 \%$, and ALP response $>50 \%$ was $29 \%$.

Patients completing $\geq 5$ cycles had longer mOS than those completing $<5$ cycles (16.2 vs. 5.9 months; $\mathrm{p}<0.0001$ ) (Fig. 2 ). Notably, only 48 patients (52.7\%) completed $\geq 5$ cycles of Ra223 in this study (72.8\% in ALSYMPCA).

Patients in the group that did not complete five cycles of Ra223 had a lower baseline hemoglobin (114 vs. 120;

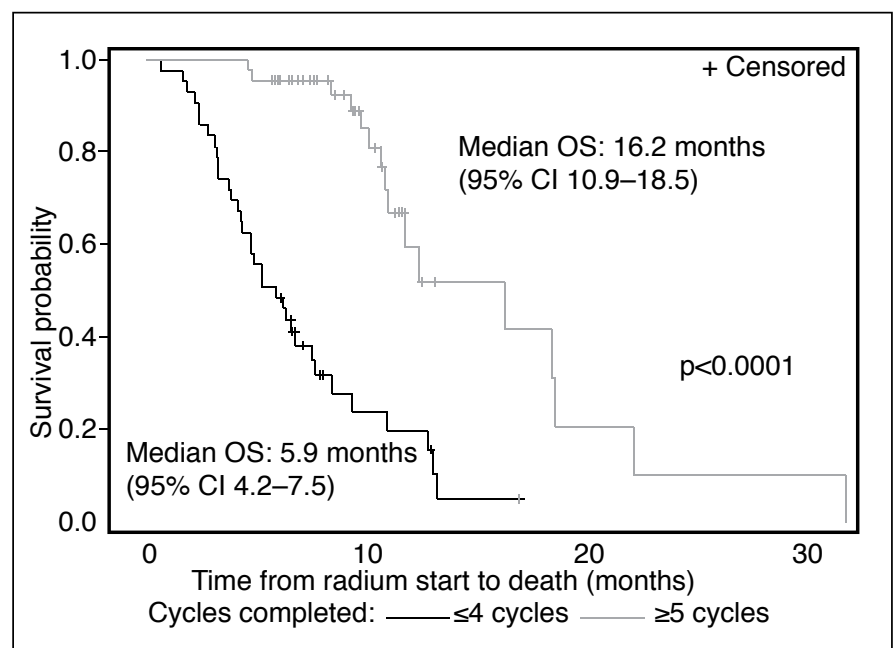

Fig. 2. Median overall survival (OS) compared between patients completing $>5$ vs. $<5$ cycles of Ra223. Cl: confidence interval. 
$p=0.03)$ and a higher baseline ALP ( $p=0.05)$ level (Table 1). Logistic regression results indicate that patients in the higherrisk baseline ALP and hemoglobin group (high baseline ALP $[\geq 220 \mathrm{IU} / \mathrm{L}]$ and low hemoglobin $[\leq 118 \mathrm{~g} / \mathrm{L}]$ ) had significantly higher odds of not completing therapy compared to patients in the lower-risk group (lower baseline ALP $[<220 \mathrm{IU} / \mathrm{L}]$ and higher hemoglobin [>118 g/L]) and intermediate-risk group (baseline ALP $[<220 \mathrm{IU} / \mathrm{L}]$ and hemoglobin $[\leq 118]$ or baseline ALP $[\geq 220 \mathrm{IU} / \mathrm{L}]$ and hemoglobin $[>118])(\mathrm{OR} 3.85 ; p=0.02$ and OR 3.82; $p=0.02$, respectively). There was no difference in the proportion of cases with an ECOG performance status greater than 2, an elevated lactate dehydrogenase (LDH), or extent of metastatic disease. The median number of previous systemic treatments was not significantly different between those completing $\geq 5$ cycles (median 2 , range $0-5$ ) vs. those completing $<5$ cycles (median 2 , range $0-5)(p=0.21$ ).

Table 2 describes PSA and ALP response rates for patients completing $\geq 5$ vs. $<5$ cycles. PSA response rate $>30 \%$ decline was greater in the group completing $\geq 5$ cycles $(33.3 \%$ vs. $7.0 \% ; p=0.002)$. PSA response rate $>50 \%$ decline was also higher in this group $(16.7 \%$ vs. $4.7 \% ; p=0.10)$, although not statistically significant (Fig. 3).

A lower proportion of patients in the group completing $\geq 5$ cycles had an ALP response $>30 \%$ ( $66.7 \%$ vs. $34.9 \%$; $\mathrm{p}=0.03)$. This held true for ALP response rate $>50 \%$ as well (37.5\% vs. $18.6 \%$; $p=0.046$ ) (Fig. 4).

The reasons for premature discontinuation are outlined in Table 3. Of note, $27.9 \%$ of patients prematurely discontinued Ra223 potentially due to drug-related side effects and $57.4 \%$ of patients prematurely discontinuing due to disease progression. The side effect profile was consistent with that seen in the ALSYMPCA trial, particularly since determination of whether the discontinuations in the current study related to low platelet and hemoglobin levels were due to disease progression or true drug-related side effect was difficult to determine.

\section{Discussion}

This retrospective study was performed to determine whether the findings in the ALSYMPCA trial were generalizable

\begin{tabular}{lcccc}
\hline \multicolumn{5}{l}{ Table 2. PSA and ALP response rates } \\
\hline Response rates & $\begin{array}{c}\text { All patients } \\
\text { (n=91) }\end{array}$ & $\begin{array}{c}\text { Completion } \\
\text { of }<5 \text { Ra223 } \\
\text { cycles }(\mathbf{n = 4 3 )}\end{array}$ & $\begin{array}{c}\text { Completion } \\
\text { of }>\mathbf{5} \text { Ra223 } \\
\text { cycles (n=48) }\end{array}$ & p \\
\hline $\begin{array}{l}\text { PSA response } \\
>30 \%, n(\%)\end{array}$ & $19(20.9)$ & $3(7.0)$ & $16(33.3)$ & 0.002 \\
$\begin{array}{l}\text { PSA response } \\
>50 \%, n(\%)\end{array}$ & $10(11.0)$ & $2(4.7)$ & $8(16.7)$ & 0.10 \\
$\begin{array}{l}\text { ALP response } \\
>30 \%, n(\%)\end{array}$ & $47(51.7)$ & $15(34.9)$ & $32(66.7)$ & 0.03 \\
$\begin{array}{l}\text { ALP response } \\
>50 \%, n(\%)\end{array}$ & $26(28.6)$ & $8(18.6)$ & $18(37.5)$ & 0.046 \\
\hline ALP: alkaline phosphatase; PSA: prostate-specific antigen. & & \\
\hline
\end{tabular}

to a population level in the contemporary era of having abiraterone and enzalutamide available, and to identify factors associated with completion of therapy. Such data could help inform practitioners about the probability of treatment completion and to identify which patients may need to be monitored more closely.

Compared to the ALSYMPCA study, patients in the present series were more heavily pre-treated, particularly with abiraterone and or enzalutamide, had a shorter median survival (11.7 months compared to 14.9 months), but had similar PSA (R30\% of $21 \%$ compared to $16 \%$ ) and ALP (R30\% 52\% compared to 47\%) response rates.

The results of this study demonstrate that therapy completion of five or more cycles was associated with a longer mOS and more frequent PSA and ALP responses. Patients that did not complete five or more cycles of Ra223 generally had higher ALP and lower hemoglobin levels at baseline. Elevated alkaline phosphatase and low hemoglobin levels are recognized poor prognostic factors for survival. ${ }^{7,10}$ It might be expected that poor prognosis patients would be less likely to respond or complete therapy; however other poor prognostic factors, like LDH and performance status, were not different between the two patient groups.

Patients in the higher-risk risk baseline ALP and hemoglobin group, who had a combination of an ALP $\geq 220$ (the usual upper limit of normal) and a hemoglobin of $\leq 118$, had significantly 3.85 (3.82) times higher odds of not completing $\geq 5$ cycles, compared to patients in the lower (intermediate) group. Thus, the higher ALP and lower hemoglobin may be specifically reflecting a higher burden of bone metastases and propensity to bone marrow toxicity, as almost $30 \%$ of patients discontinued because of toxic effects. Marrow toxicity in particular is often hard to clearly delineate as disease progression or drug effect, as both can lead to decreasing blood counts, but in either case, may make further doses ill-advised. Of the 12 patients that were scored as having stopped radium due to marrow toxicity, nine $(75 \%)$ had coincident rise in PSA at the time of discontinuation, suggesting the underlying cause of decline in hematological

Table 3. Reasons for premature discontinuation of Ra223 (n=54)

\begin{tabular}{lc}
\hline Reasons for Ra223 discontinuation & $\mathbf{n}(\%)$ \\
\hline Gastrointestinal side effects & $3(5.6)$ \\
Anemia & $9(16.7)$ \\
Thrombocytopenia & $3(5.6)$ \\
Disease progression* & $31(57.4)$ \\
Other** & $8(14.8)$ \\
\hline *Disease progression included clinical biochemical, or radiological progression. Clinical \\
progression included skeletal related events (i.e., pathological fractures, spinal cord \\
compression, necessity for radiation therapy or surgery to the bone), and bone pain, or \\
fatigue deemed due to cancer progression. **Other included the following: exacerbation \\
of osteomyelitis, cardiac-related death, switch to clinical trial, hypercalcemia, renal function \\
decline, exacerbation of multiple prior comorbidities, worsening of known aortic stenosis, \\
and lack of Ra223.
\end{tabular}




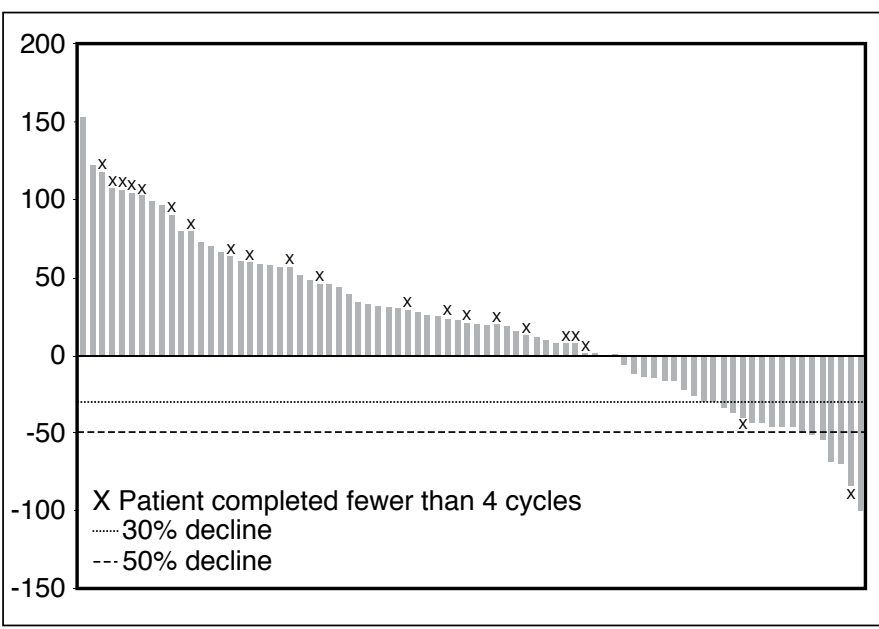

Fig. 3. Waterfall plot of maximum prostate-specific antigen (PSA) decline up to Cycle 4.

function may have been related to disease progression rather than drug toxicity.

Other studies have had similar findings to ours. Recent work by McKay and colleagues ${ }^{9}$ has suggested that receipt of less than five cycles of Ra223 was also associated with poor prognosis features and baseline marrow compromise: baseline hemoglobin levels $\geq$ lower limit of normal (LLN), prior sipuleucel-T therapy, and ANC $\geq$ LLN in multivariable analysis. Saad and colleagues ${ }^{10}$ investigated efficacy data on mCRPC patients receiving Ra223 in an international, early access, open-label, single-arm phase $3 b$ trial. They determined that mOS was longer for patients with a baseline hemoglobin level $\geq 100 \mathrm{mg} / \mathrm{L}$, baseline ALP levels < upper limit of normal, ECOG status of 0 , and for those with no reported baseline pain. Several of these and/or other factors have been studied with various agents in MCRPC and have similarly shown to be effective prognostic markers. ${ }^{11-15}$

Obtaining results from real-world settings is an informative aspect of retrospective data and strength of this study. Another important strength is that these results reflect patients treated across several oncology centres, which increases the generalizability of results. The study is limited by its retrospective nature, small patient numbers, and limited followup. Although we are unable to identify a control group for robust statistical comparison, our group has previously investigated the outcomes of a similar group of patients (those with symptomatic bone metastases requiring palliative radiotherapy to bone $[P R T B])$, and the survival of such patients after first course of PRTB was 8.5 months. ${ }^{16}$ In addition, for a sense of context of the proportion of patients receiving Ra223 relative to those that need it, we were able to assess the number of symptomatic patients receiving PRTB in a one-year period for the Vancouver Cancer Centre catchment area from the previous study. ${ }^{16}$ In the three years preceding the availability of Ra223, 40-56 patients received PRTB prior to eventual death from prostate cancer per year; whereas, in the first full year of

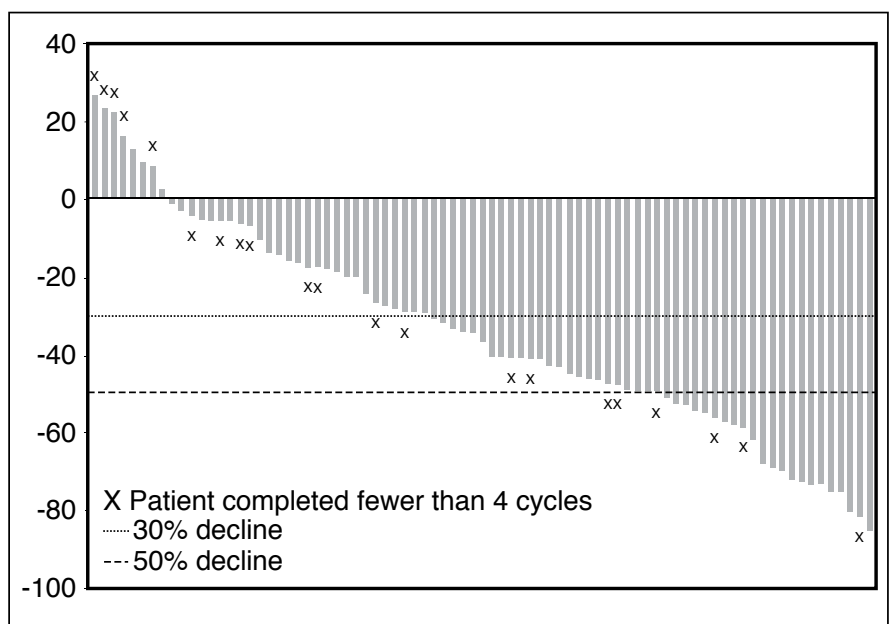

Fig. 4. Waterfall plot of maximum alkaline phosphatase (ALP) decline up to Cycle 4.

Ra223 availability in the Vancouver Cancer Centre catchment area in the current study, 15 patients received Ra223, suggesting a radium utilization rate of approximately one-third relative to symptomatic patients referred for and receiving PRTB.

\section{Conclusion}

This data reflects a population-based experience with Ra223 in mCRPC. It demonstrates less cycle completion, shorter surviv$\mathrm{al}$, and more lines of prior therapy relative to the randomized trial data, and that higher ALP and lower hemoglobin levels were factors associated lower rate of treatment completion.

Competing interests: Dr. Parimi has received speaker honoraria from and participated in clinical trials supported by Astellas and Janssen. Dr. Alexander has received speaker honoraria from Bayer. Dr. McKenzie has been an advisor for and participated in clinical trials supported by Janssen; and has received honoraria from Amgen and Bayer. Dr. Bachand has received speaker honoraria from Bayer. Dr. Chi has received grant and honoraria from Astellas, Bayer, Essa, Janssen, and Sanofi; and has participated in clinical trials supported by Astellas, Bayer, Eli Lilly, Janssen, Merck, and Novartis. Dr. Tyldesley has received speaker honoraria from Bayer. The remaining authors report no competing personal or financial interests.

This paper has been peer-reviewed.

\section{References}

1. Beer TM, Armstrong AJ, Rathkopf DE, et al. Enzalutamide in metastatic prostate cancer before chemotherapy. N Engl J Med 2014;371:424-33. https://doi.org/10.1056/NEJMoa 1405095

2. Ryan CJ, Smith MR, de Bono JS, et al. Abiraterone in metastatic prostate cancer without previous chemotherapy. N Engl J Med 2013;368:138-48. https://doi.org/10.1056/NEJMoal209096

3. Scher HI, Fizazi K, Saad F, et al. Increased survival with enzalutamide in prostate cancer after chemotherapy. N Engl J Med 2012;367:1187-97. https://doi.org/10.1056/NEJMoal207506

4. De Bono IS, Logothetis $\mathrm{CJ}$, Molina A, et al. Abiraterone and increased survival in metastatic prostate cancer. N Engl J Med 2011;364:1995-2005. htrps://doi.org/10.1056/NEJMoa1014618 


\section{${ }^{8} \mathrm{XGEVA}$ (denosumab)}

Indication and clinical use:

-XGEVA is indicated for reducing the risk of developing skeletal-related events (SREs) in patients with bone metastases from breast cancer, prostate cancer, non-small cell lung cancer, and other solid tumours.

- Not indicated for reducing the risk of developing skeletal-related events in patients with multiple myeloma.

- Not indicated for reducing the risk of developing skeletal-related events in pediatric patients.

\section{Contraindication:}

-XGEVA is contraindicated in patients with pre-existing hypocalcemia, which must be corrected prior to initiating therapy.

\section{Most serious warnings and precautions:}

Osteonecrosis of the jaw (ONJ): In clinical trials, the incidence of ONJ was higher with longer duration of exposure. In patients with risk factors for ONJ, an individual risk/benefit assessment should be performed before initiating therapy with XGEVA. An oral exam should be performed and a dental exam with appropriate preventive dentistry is recommended prior to treatment with XGEVA, especially in patients with risk factors for $0 \mathrm{NJ}$. Avoid invasive dental procedures while receiving XGEVA. In patients who develop ONJ during treatment with XGEVA, a temporary interruption of treatment should be considered based on individual risk/benefit assessment until the condition resolves.

Other relevant warnings and precautions:

- Do not use concurrently with Prolia ${ }^{\circledR}$

- Do not use concurrently with bisphosphonates

- Hypocalcemia has been reported (including severe symptomatic hypocalcemia and fatal cases). Monitor calcium prior to the initial dose, within two weeks after the initial dose, and if suspected symptoms of hypocalcemia occur. Administer adequate calcium, vitamin $D$, and magnesium, as necessary. If hypocalcemia occurs while receiving XGEVA, additional short-term calcium supplementation and additional monitoring may be necessary.

- Caution on risk of hypocalcemia and accompanying increases in parathyroid hormone in patients with renal impairment

- Clinically significant hypercalcemia has been reported in XGEVA-treated patients with growing skeletons weeks to months following treatment discontinuation. Monitor patients for signs and symptoms of hypercalcemia and treat appropriately.

- Skin infections

- Hypersensitivity reactions including anaphylaxis

- Atypical femoral fractures

- Not recommended for use in pregnant women. Women should not become pregnant during treatment and for at least 5 months after the last dose of XGEVA.

- For nursing women, it is not known whether XGEVA is excreted into human milk.

For more information:

Please consult the Product Monograph at

http://www.amgen.ca/Xgeva_PM.pdf for important information relating to adverse reactions, drug interactions, and dosing that have not been discussed here.

The Product Monograph is also available by calling Amgen Medical Information at 1-866-502-6436.

\section{Fizazi et al. study ${ }^{2}$}

Phase 3, randomized, double-blind, double-dummy, active-controlled study. Patients with castrate-resistant prostate cancer and bone metastases $(n=1901)$ received either $120 \mathrm{mg}$ XGEVA ${ }^{\circledast}$ SC Q4W (once every 4 weeks) ( $\left.n=950\right)$ or $4 \mathrm{mg}$ zoledronic acid IV Q4W $(n=951)$. The primary outcome measure was to demonstrate non-inferiority of time to first on-study SRE as compared to zoledronic acid. The secondary outcome measures were superiority of time to first on-study SRE and superiority of time to first and subsequent SRES. An SRE is defined as any of the following: pathologic fracture, radiation therapy to bone, surgery to bone or spinal cord compression.

\section{References:}

1. XGEVA ${ }^{\circledast}$ Product Monograph, Amgen Canada, 2016.

2. Fizazi K, et al. Denosumab versus zoledronic acid for treatment of bone metastases in men with castration-resistant prostate cancer: a randomized, double-blind study. Lancet. 2011;377(9768):813-822.
5. Tannock IF, de Wit R, Berry WR, et al. Docetaxel plus prednisone or mitoxantrone plus prednisone for advanced prostate cancer. N Engl J Med 2004;351:1502-12. https://doi.org/10.1056/ NEJMoa040720

6. De Bono JS, Oudard S, Ozguroglu $M$, et al. Prednisone plus cabazitaxel or mitoxantrone for metastatic castration-resistant prostate cancer progressing after docetaxel treatment: A randomized, open-label trial. Lancet 2010;376:1147-54. https://doi.org/10.1016/S0140-6736(10)61389-X

7. Parker $C$, Nilsson $S$, Heinrich D, et al. Alpha emitter radium-223 and survival in metastatic prostate cancer. N Engl J Med 2013;369:213-23. https://doi.org/10.1056/NEJMoal213755

8. Eisenhaver $E A$, Therasse $P$, Bogaerts J, et al. New response evaluation criteria in solid tumours: Revised RECIST guideline (version 1.1). Eur J Cancer 2009;45:228-47. https://doi.org/10.1016/i. ejca.2008.10.026

9. McKay RR, Jacobus S, Fiorillo M, et al. Radium-223 utilization in clinical practice and variables associated with completion of therapy. Clin Genitourin Cancer 2017;15:e289-e98. https://doi.org/10.1016/i. clgc.2016.08.015

10. Saad F, Carles J, Gillessen S, et al. Radium-223 and concomitant therapies in patients with metastatic castration-resistant prostate cancer: An international, early access, open-label, single-arm phase $3 \mathrm{~b}$ trial. Lancet Oncol 2016;17:1306-16. https://doi.org/10.1016/S1470-2045(16)30173-5

11. Armstrong AJ, Garrett-Mayer $E$, de Wit $R$, et al. Prediction of survival following first-line chemotherapy in men with castration-resistant metastatic prostate cancer. Clin Cancer Res 2010;16:203-11. https://doi.org/10.1158/1078-0432.CCR-09-2514

12. Fizazi K, Massard C, Smith M, et al. Bone-related parameters are the main prognostic factors for overall survival in men with bone metastases from castration-resistant prostate cancer. Eur Urol 2015;68:42-50. https://doi.org/10.1016/i.eururo.2014.10.001

13. Azad AA, Eigl BJ, Leibowitz-Amit $R$, et al. Outcomes with abiraterone acetate in metastatic castrationresistant prostate cancer patients who have poor performance status. Eur Urol 2014;1-7.

14. Etchebehere EC, Milton DR, Araujo JC, et al. Factors affecting 223Ra therapy:Clinical experience after 532 cycles from a single institution. Eur J Nucl Med Mol Imaging 2016;43:8-20. https://doi.org/10.1007/ s00259-015-3185-4

15. Chi KN, Kheoh T, Ryan CJ, et al. A prognostic index model for predicting overall survival in patients with metastatic castration-resistant prostate cancer treated with abiraterone acetate after docetaxel. Ann Oncol 2016;27:454-60. https://doi.org/10.1093/annonc/mdv594

16. Cho J, Pickles T, Bachand F, et al. Palliative radiotherapy for bone metastases in patients dying of prostate cancer: The British Columbia experience. CARO 2016. Radioth Oncol 2016:120: S89-90. https://doi.org/10.1016/S0167-8140(16)33645-3

Correspondence: Dr. Scott Tyldesley, Radiation Oncology, British Columbia Cancer Agency, Vancouver, BC, Canada; styldes|@bccancer.bc.ca

\section{Oncology}

